\section{Sentimento de felicidade em idosos: uma abordagem epidemiológica, ISA-Camp 2008}

\author{
Happiness in the elderly: an epidemiological \\ approach in the ISA-Camp 2008 study
}

Abstract

${ }^{1}$ Faculdade de Ciências Médicas, Universidade Estadual de Campinas, Campinas, Brasil.

2 Instituto de Saúde, Secretaria de Estado da Saúde de São Paulo, São Paulo, Brasil.

Correspondência M. G. Lima

Departamento de Saúde Coletiva, Faculdade de Ciências Médicas, Universidade Estadual de Campinas.

Rua Tessália Vieira de Camargo 126, Campinas, SP 13083-887, Brasil. margareth.guimaraes@ yahoo.com.br
The objective was to identify factors associated with happiness in the elderly. A cross-sectional, population-based study was conducted in 1,431 elderly under the ISA-Camp 2008 project. The survey used a two-stage probabilistic cluster sample. Prevalence of happiness was measured over time according to socio-demographics variables, health behaviors, and health conditions. High prevalence of happiness was associated with: marital status (married), active working, activity and insufficient leisure-time activity, occasional consumption of alcoholic beverages, daily consumption of fruit, vegetables, and leafy vegetables, normal body mass index, and sleeping less than 10 hours/night and sleeping well. The highest prevalence of long-term happiness was observed among elderly with no reported illness, with better self-rated health, and with less disability. Happiness was strongly related to health indicators, suggesting the adequacy of complementary use of this indicator for evaluating health promotion programs in the elderly.

Health of the Elderly; Health Surveys; Happiness

\author{
Margareth Guimarães Lima 1 \\ Marilisa Berti de Azevedo Barros 1 \\ Maria Cecilia Goi Porto Alves 2
}

\section{Introdução}

Elucidar as relações entre o sentimento de felicidade e as condições de saúde é tarefa que precisa ser empreendida, visto que o bem-estar de indivíduos e populações é a meta final almejada pelas políticas e ações de saúde 1,2. O modelo de saúde proposto por Evans \& Stoddart 1 baseia-se na compreensão de que fatores ambientais, sociais e genéticos imprimem padrões de respostas biológicas e comportamentais, que incidem sobre o estado de saúde e a capacidade funcional na medida em que podem propiciar a ocorrência de doenças. Estas dimensões da saúde acabam, por sua vez, influenciando fortemente o nível de bem-estar de indivíduos e populações.

É importante considerar a perspectiva objetiva do bem-estar, como as condições de vida consumo, arte, conforto e comodidades. Estes fatores podem ser medidos por meio de indicadores de renda, educação, nível de desemprego expectativa de vida e outros. Por outro lado, considera-se a dimensão subjetiva do bem-estar e da qualidade de vida. Nessa perspectiva, a medida se dá com base na avaliação do próprio sujeito sobre sua satisfação com a vida ${ }^{3}$.

Os estudos sobre o bem-estar subjetivo se desenvolveram principalmente no final do século $\mathrm{XX}$, quando a psicologia começa a se preocupar com o entendimento das emoções positivas, em vez de focar a atenção apenas na doença mental. E foi a partir da década de 70 que cresceu o nú- 
mero de estudos sobre a felicidade e o bem-estar subjetivo 4,5 .

Segundo Diener 6, o bem-estar subjetivo é resultante do grau de satisfação com a vida em relação a diversificados domínios, especialmente os do trabalho e da família, e do balanço entre os afetos, positivos e negativos. Afetos são sentimentos de prazer ou desprazer que se constituem a partir dos humores (sentimento mais duradouro) e das emoções (sentimento mais breve e intenso) ${ }^{5}$. Veenhoven 7 define felicidade como o grau em que um indivíduo avalia, de maneira favorável, a qualidade do conjunto dos aspectos e dimensões de sua vida.

Os conceitos de felicidade e satisfação com a vida, embora muito interligados, diferem em aspectos relacionados à cognição: a satisfação com a vida enfatiza o processo de avaliação, enquanto o sentimento de felicidade, mesmo com algum componente cognitivo, desenvolver-se-ia com maior peso a partir das emoções 7,8. Apesar das particularidades, existe uma forte inter-relação entre os conceitos de bem-estar subjetivo, felicidade e satisfação com a vida. Nota-se, porém, que não há um consenso para a utilização destes termos 5 .

O sentimento de felicidade tem, recentemente, recebido atenção no campo da investigação científica da saúde 9. Estudos longitudinais têm apontado a importância da felicidade e do otimismo no aumento da longevidade $10,11,12$, na proteção a morbidades 13,14 e incapacidades 15 e na tendência à adoção de comportamentos saudáveis 11,16. Pesquisas evidenciam que o sentimento de felicidade está fortemente associado com a autoestima, com o conforto domiciliar, com o suporte e a participação social, sendo influenciado também pela situação conjugal 17,18,19,20.

São poucos os estudos voltados a analisar a relação do sentimento de felicidade com as condições de saúde. No entanto, duas pesquisas realizadas com adultos no Brasil apontaram significativa associação da autoavaliação de saúde e da prevalência de doenças crônicas com a felicidade 20,21.

A relação da felicidade com os comportamentos de saúde nos idosos também não é muito conhecida. Segundo alguns autores, os afetos positivos podem levar à maior adesão a comportamentos saudáveis, incluindo a prática de atividade física, os cuidados com a alimentação, a cessação do tabagismo e o controle do peso corporal. Deve-se ressaltar, contudo, que as evidências dessas associações ainda são escassas 9,11,16,22.

Considerando a necessidade do desenvolvimento e estudo de indicadores de bem-estar e da melhor compreensão da relação desses indicado- res, particularmente a felicidade, com condições e determinantes da saúde, este estudo tem como objetivo detectar os fatores demográficos, socioeconômicos, de comportamentos relacionados à saúde e de condições de saúde, que se associam ao sentimento de felicidade na população idosa.

\section{Métodos}

O estudo é do tipo transversal, de base populacional, que utilizou dados do inquérito de saúde realizado na cidade de Campinas (ISA-Camp 2008). Os dados foram coletados em 2008 e início de 2009, na área urbana de Campinas, São Paulo, por meio de um questionário pré-codificado, aplicado por entrevistadores treinados.

A amostragem do ISA-Camp 2008 foi por conglomerados e em dois estágios: setores censitários e domicílios. Foram selecionados 50 setores com probabilidade proporcional ao número de domicílios.

Foi definido um tamanho mínimo de amostra de mil pessoas para cada domínio de idade (10 a 19 anos; 20 a 59 anos e 60 anos e mais); as amostras para cada domínio foram sorteadas de forma independente. Esse número de entrevistas assegura estimativas de proporção de 0,50 , com erro de até cinco pontos percentuais, com intervalos de $95 \%$ de confiança (IC95\%), considerando um efeito de delineamento de 2. Para encontrar esse tamanho de amostra em cada grupo de idade, foram selecionados 1.672, 522, e 3.092 domicílios, respectivamente para os adolescentes, os adultos e os idosos. Considerando uma possível perda de $20 \%$ por domicílio fechado ou recusa, o número de domicílios selecionados foi aumentado para 2.250, 700 e 3.900, respectivamente. Foram entrevistados todos os moradores do grupo de idade sorteado para o domicílio.

Este estudo analisou apenas as pessoas com 60 anos ou mais de idade que responderam pessoalmente à entrevista, inclusive à questão sobre o sentimento de felicidade nas últimas quatro semanas. Foram excluídos os idosos que não conseguiram responder ao questionário devido a incapacidades graves, como, por exemplo, doença senil, totalizando 1.431 idosos.

A variável dependente do estudo foi o sentimento de felicidade nas últimas quatro semanas, extraída da questão 09h do The Medical Outcomes Study 36-Item Short-Form Health Survey (SF-36) 23: "Por quanto tempo, durante as últimas quatro semanas, você tem se sentido feliz?”. As respostas foram categorizadas em: 0 (todo o tempo) e 1 (a maior parte, alguma parte do tempo, pequena parte do tempo, ou nunca). A categoria "por todo o tempo" foi considerada como 
referência, em virtude do objetivo de estudar o bem-estar como indicador positivo.

As variáveis independentes analisadas foram: sexo (masculino e feminino); faixa etária (60 a 69, 70 a 79 e 80 anos e mais); situação conjugal (casado/vive junto, solteiro/separado, viúvo); religião (católica, evangélica e outras); cor da pele (branca, preta/parda/amarela); número de moradores no domicílio (1, 2, 3, 4 ou mais); escolaridade em anos de estudo (0 a 3, 4 a 8, 9 e mais); renda mensal per capita em salários mínimos (menos de 1, 1 a 3, mais de 3); ocupação (trabalha, aposentado(a), dona de casa, outras); número de equipamentos no domicílio (5 ou menos, 6 a 11, 12 ou mais); posse referida de carro, computador, Internet, telefone fixo ou celular (sim ou não); tabagismo (fumante, não fumante, ex-fumante); ingestão de bebida alcoólica (não ingere, ingere menos de uma vez por semana, ingere uma ou mais vezes por semana); uso abusivo de bebida alcoólica (presente ou ausente) - avaliado pelo Alcohol Use Disorders Identification Test (AUDIT), considerando fazer uso abusivo os idosos com oito ou mais pontos positivos em uma escala de 0 a 40 24; atividade física no lazer (sedentário, insuficientemente ativo, ativo) - pessoas que realizaram pelo menos 150 minutos de atividade física por semana, em pelo menos três dias da semana, foram considerados ativos, e os que praticaram alguma atividade física no lazer, sem atingir esses índices, foram considerados insuficientemente ativos; ingestão de frutas, legumes e verduras todos os dias (sim ou não); índice de massa corporal (IMC), proveniente de informações de peso e altura autorreferidos (baixo peso: menor que $22 \mathrm{~kg} / \mathrm{m}^{2}$, eutrófico: $22-27 \mathrm{~kg} / \mathrm{m}^{2}$, sobrepeso: 28 $29 \mathrm{~kg} / \mathrm{m}^{2}$, obesidade: $30 \mathrm{~kg} / \mathrm{m}^{2}$ ou mais); duração do tempo de sono, considerando dias da semana e de final de semana ( 5 horas ou menos, 6 horas, 7 a 8, 9, 10 horas ou mais); número de doenças crônicas, considerando hipertensão, diabetes, doença do coração, tumor ou câncer, reumatismo/artrite/artrose, asma/bronquite/enfisema, tendinite/LER/DORT, problemas circulatórios (nenhuma, 1, 2 ou 3, 4 ou mais); qualidade do sono, obtida pela questão: "Nos últimos 30 dias você dormiu mal?" (não ou sim); autoavaliação do estado de saúde (excelente/muito boa, boa e ruim/muito ruim); incapacidade funcional, definida com base nas questões 03g, 03h, 03i e 03j, obtidas do SF-36 23 (grave: dificuldade em tomar banho ou vestir-se; moderada: dificuldade de andar um ou vários quarteirões; leve: dificuldade de andar mais do que $1 \mathrm{~km}$; ausente: nenhuma destas dificuldades).

Foram estimadas as prevalências do sentimento de felicidade por todo o tempo, nas últimas quatro semanas, e testada sua associação com variáveis demográficas, socioeconômicas, de comportamento e de estado de saúde, por meio do teste do qui-quadrado de Pearson. Razões de prevalências (RP), brutas e ajustadas, e respectivos IC95\% foram estimados por meio de regressão de Poisson, simples e múltipla. As variáveis que apresentaram valor de $\mathrm{p}<0,20$ na análise simples compuseram o modelo múltiplo, e apenas aquelas com valor de $\mathrm{p}<0,05$ permaneceram no modelo.

O efeito do desenho amostral complexo foi considerado em todas as análises realizadas, utilizando-se o software Stata 11.0 (Stata Corp., College Station, Estados Unidos). Este estudo foi aprovado pelo comitê de ética da Faculdade de Ciências Médicas da Universidade Estadual de Campinas, sob parecer no. 079/2007.

\section{Resultados}

Entre os domicílios sorteados para obter a amostra de idosos, houve uma perda de 14,2\%. Destas, $6,1 \%$ devido à recusa; $3,1 \%$ porque o indivíduo não foi encontrado no domicílio; $5 \%$ por outros motivos. Entre os idosos encontrados nos domicílios, houve 5,5\% de recusa e $6,9 \%$ de perdas por outros motivos. Dos idosos entrevistados, 88 não tiveram condições físicas e/ou mentais para responderem seu próprio questionário (o qual, nesta situação, foi respondido por familiar ou cuidador), não tendo sido, por esse motivo, incluídos nesta análise. Portanto, a amostra do presente estudo foi constituída por 1.431 indivíduos idosos.

A idade média da população estudada foi de 69,5 anos (IC95\%: 68,8-70,1); 57,1\% (IC95\%: 54,6-59,6) eram pessoas do sexo feminino; $40,5 \%$ tinham de quatro a oito anos de estudo e $39 \%$ viviam com menos de um salário mínimo de renda familiar per capita. Dos idosos estudados, $28,2 \%$ eram viúvos e $21,1 \%$ tinham ainda alguma ocupação remunerada.

As prevalências do sentimento de felicidade nas últimas quatro semanas foram: todo o tempo, 35,4\% (IC95\%: 30,2-40,6); a maior parte do tempo, 41,8\% (IC95\%: 37,7-45,8); alguma parte do tempo, 14,5\% (IC95\%: 11,5-17,4); pequena parte do tempo, 7,2\% (IC95\%: 5,6-8,7); nunca, 0,01\% (IC95\%: 0,004-0,015).

Como se pode observar nas Tabelas 1 e 2, a prevalência de idosos que se declararam felizes por todo o tempo durante as últimas quatro semanas foi significativamente maior nos homens e nos mais jovens, nos casados em relação aos viúvos, naqueles com maior nível de escolaridade e de renda, nos que ainda trabalham e nos idosos que possuem carro e acesso à Internet. No mo- 
Prevalência e razão de prevalências (RP) do sentimento de felicidade por todo o tempo, segundo fatores sociodemográficos, na população idosa de Campinas, São Paulo, Brasil. ISA-Camp, 2008.

\begin{tabular}{|c|c|c|c|c|c|}
\hline Variável & n (\%) & $\begin{array}{c}\text { Prevalência de pessoas que se } \\
\text { declaram felizes por todo o } \\
\text { tempo (IC95\%) }\end{array}$ & Valor de $p$ * & $\mathrm{RP} * \star(I C 95 \%)$ & Valor de $p$ \\
\hline Sexo & & & 0,001 & & \\
\hline Feminino & $852(57,1)$ & $31,2(26,2-36,8)$ & & 1,00 & \\
\hline Masculino & $579(42,8)$ & $40,9(34,9-47,2)$ & & $1,31(1,15-1,48)$ & $<0,001$ \\
\hline Idade (anos) & & & 0,015 & & \\
\hline $60-69$ & $799(55,8)$ & $38,8(33,1-44,9)$ & & 1,00 & \\
\hline $70-79$ & $470(32,7)$ & $30,2(24,2-37,1)$ & & $0,77(0,65-0,93)$ & 0,007 \\
\hline 80 e mais & $162(11,3)$ & $33,2(25,6-41,9)$ & & $0,85(0,66-1,09)$ & 0,216 \\
\hline Situação conjugal & & & $<0,001$ & & \\
\hline Casado/Vive junto & $812(57,5)$ & $39,7(33,7-46,0)$ & & 1,00 & \\
\hline Solteiro/Separado & $203(14,1)$ & $34,9(27,3-43,3)$ & & $0,87(0,69-1,11)$ & 0,285 \\
\hline Viúvo & $416(28,2)$ & $26,9(21,2-33,4)$ & & $0,67(0,56-0,80)$ & $<0,001$ \\
\hline Religião & & & 0,563 & & \\
\hline Católica & $950(66,5)$ & $34,6(29,5-40,1)$ & & 1,00 & \\
\hline Evangélica & $318(21,6)$ & $34,8(26,8-43,7)$ & & $1,00(0,80-1,25)$ & 0,965 \\
\hline Outras ou sem religião & $162(11,7)$ & $40,2(29,3-52,1)$ & & $1,15(0,86-1,54)$ & 0,308 \\
\hline Cor & & & 0,465 & & \\
\hline Branca & $1.083(76,3)$ & $36,0(30,9-41,5)$ & & 1,00 & \\
\hline Preta/Amarela/Outras (não branca) & $346(23,7)$ & $33,1(25,4-41,9)$ & & $0,92(0,72-1,15)$ & 0,473 \\
\hline Número de moradores no domicílio & & & 0,138 & & \\
\hline 4 ou mais & $355(24,3)$ & $32,8(26,1-40,2)$ & & 1,00 & \\
\hline 3 & $283(19,9)$ & $33,1(27,1-39,7)$ & & $1,01(0,80-1,27)$ & 0,929 \\
\hline 2 & $551(38,9)$ & $39,4(32,4-46,9)$ & & $1,20(0,94-1,53)$ & 0,137 \\
\hline 1 & $242(16,7)$ & $32,6(26,5-39,4)$ & & $0,99(0,78-1,27)$ & 0,973 \\
\hline
\end{tabular}

IC95\%: intervalo de 95\% de confiança.

* Teste de qui-quadrado;

** Calculada por meio de regressão de Poisson.

delo de regressão múltipla, que incluiu as variáveis demográficas e socioeconômicas (Tabela 3), observou-se que fatores como viuvez e não trabalhar associaram-se inversamente com o sentimento de felicidade nos idosos estudados, mesmo após ajuste por idade, sexo e escolaridade.

Segundo comportamentos relacionados à saúde, a análise ajustada indica que os idosos que ingerem bebida alcoólica ocasionalmente os que são ativos ou insuficientemente ativos no lazer e os que consomem verduras, legumes e frutas todos os dias apresentaram a maior prevalência de felicidade por todo o tempo nas últimas quatro semanas. Os idosos com IMC igual ou superior a $30 \mathrm{~kg} / \mathrm{m}^{2}$, os que dormem 10 horas ou mais por dia ou que referem dormir mal apresentaram as menores prevalências de felicidade por todo o tempo (Tabela 4).
Na Tabela 5, com ajustes pelas variáveis demográficas e socioeconômicas, verifica-se forte associação de indicadores das condições de saúde com o tempo de felicidade. Os resultados apontam gradientes na associação do sentimento de felicidade com o número de morbidades, com a qualidade da saúde autoavaliada e com a presença e nível de incapacidades. As maiores prevalências do sentimento de felicidade por todo o tempo encontram-se nos idosos que não apresentam doenças crônicas ( $\mathrm{RP}=1,83$ ) ou com apenas uma doença $(\mathrm{RP}=1,56)$, em relação aos que apresentam quatro ou mais. $\mathrm{O}$ segmento que considera sua saúde como boa $(\mathrm{RP}=3,74)$ e muito boa ou excelente ( $\mathrm{RP}=5,49$ ), os idosos com incapacidade funcional leve $(1,95)$ ou aqueles que não apresentam as incapacidades estudadas $(2,52)$ encontram-se com maior prevalência de 
Tabela 2

Prevalência e razão de prevalências (RP) do sentimento de felicidade por todo o tempo, segundo fatores socioeconômicos, na população idosa de Campinas, São Paulo, Brasil. ISA-Camp, 2008.

\begin{tabular}{|c|c|c|c|c|c|}
\hline Variável & n (\%) & $\begin{array}{l}\text { Prevalência de pessoas que se declaram } \\
\text { felizes por todo o tempo (IC95\%) }\end{array}$ & $\begin{array}{l}\text { Valor de } \\
\qquad p^{*}\end{array}$ & $\mathrm{RP} * \star(I C 95 \%)$ & Valor de $p$ \\
\hline Escolaridade (anos de estudo) & & & 0,005 & & \\
\hline $0-3$ & $503(34,1)$ & $28,8(22,0-36,7)$ & & 1,00 & \\
\hline $4-8$ & $586(40,5)$ & $35,9(30,1-42,2)$ & & $1,24(0,98-1,58)$ & 0,066 \\
\hline 9 e mais & $341(25,4)$ & $43,1(36,6-49,9)$ & & $1,49(1,12-1,98)$ & 0,006 \\
\hline Renda familiar per capita (salários & & & 0,080 & & \\
\hline \multicolumn{6}{|l|}{ mínimos) } \\
\hline Menos de 1 & $571(39,0)$ & $31,5(25,2-38,5)$ & & 1,00 & \\
\hline $1-3$ & $696(48,5)$ & $37,0(30,7-43,8)$ & & $1,17(0,95-1,45)$ & 0,130 \\
\hline Mais de 3 & $164(12,3)$ & $41,3(34,7-48,2)$ & & $1,31(1,01-1,69)$ & 0,040 \\
\hline Ocupação & & & $<0,001$ & & \\
\hline Trabalha (aposentado ou não) & $294(21,1)$ & $49,1(41,8-56,4)$ & & 1,00 & \\
\hline Aposentado & $702(49,5)$ & $33,6(27,8-39,9)$ & & $0,68(0,57-0,82)$ & $<0,001$ \\
\hline Dona de casa & $390(26,1)$ & $29,3(24,0-35,4)$ & & $0,59(0,49-0,72)$ & $<0,001$ \\
\hline Outras & $44(3,0)$ & $22,3(11,3-39,1)$ & & $0,45(0,24-0,83)$ & 0,012 \\
\hline Número de equipamentos no & & & 0,376 & & \\
\hline \multicolumn{6}{|l|}{ domicílio } \\
\hline 5 ou menos & $290(19,6)$ & $31,9(21,8-42,6)$ & & 1,00 & \\
\hline $6-11$ & $666(45,9)$ & $35,3(28,8-42,5)$ & & $1,12(0,80-1,59)$ & 0,480 \\
\hline $12-14$ & $211(15,1)$ & $34,1(25,8-43,5)$ & & $1,09(0,71-1,65)$ & 0,681 \\
\hline 15 ou mais & $264(19,3)$ & $40,8(35,3-46,6)$ & & $1,30(0,93-1,82)$ & 0,114 \\
\hline \multicolumn{6}{|l|}{ Posse dos equipamentos } \\
\hline Carro & $741(52,8)$ & $38,3(33.8-44,2)$ & 0,034 & $1,23(1,00-1,50)$ & 0,042 \\
\hline Computador & $490(35,4)$ & $39,0(33,4-44,7)$ & 0,106 & $1,16(0,96-1,39)$ & 0,111 \\
\hline Internet & $402(29,1)$ & $42,5(36,3-48,9)$ & 0,006 & $1,30(1,07-1,58)$ & 0,007 \\
\hline Telefone fixo ou celular & $145(9,7)$ & $36,3(31,6-41,4)$ & 0,106 & $1,37(0,90-2,10)$ & 0,136 \\
\hline
\end{tabular}

IC95\%: intervalo de 95\% de confiança.

* Teste de qui-quadrado;

** Calculada por meio de regressão de Poisson.

felicidade por todo o tempo, em relação àqueles que se autoavaliam com a saúde ruim ou muito ruim e que apresentam incapacidade grave, respectivamente.

\section{Discussão}

Segundo Diener 4, o bem-estar subjetivo pode ser influenciado por fatores relativos à personalidade, às aspirações pessoais e aos processos de adaptação e estratégias de coping, ou seja, a maneira com que o indivíduo lida com os eventos estressantes 4,5 . As condições demográficas e socioeconômicas, bem como as condições e comportamentos de saúde, também podem explicar partes do bem-estar subjetivo ${ }^{4}$. Ressalta- se a importância de estudos sobre a relação da felicidade com os fatores que são passíveis de intervenções e mudanças.

A prevalência do sentimento de felicidade e seus fatores associados, no âmbito da saúde, constitui uma temática pouco estudada no Brasil. Rodrigues \& Silva 20 estudaram os fatores associados à felicidade em 467 adultos, na faixa etária de 15 a 66 anos, da cidade de Ribeirão Preto, São Paulo. Barata et al. 21 estudaram a autoavaliação de saúde segundo fatores de vulnerabilidade, discriminação e felicidade em 917 adultos residentes na área central da cidade de São Paulo. Joia et al. 17 avaliaram os fatores associados ao grau de satisfação com a vida em 365 idosos residentes na cidade de Botucatu, São Paulo, utilizando uma escala visual para a aferição. Guedea 
Fatores sociodemográficos e socioeconômicos associados ao sentimento de felicidade por todo o tempo, nas últimas quatro semanas: modelo final da regressão múltipla de Poisson. ISA-Camp, 2008.

\begin{tabular}{|c|c|c|}
\hline Variável & $\begin{array}{c}\text { Total } \\
\text { RP * (IC95\%) }\end{array}$ & Valor de $p$ \\
\hline \multicolumn{3}{|l|}{ Sexo } \\
\hline Feminino & 1,00 & \\
\hline Masculino & $1,13(0,94-1,36)$ & 0,168 \\
\hline \multicolumn{3}{|l|}{ Faixa etária (anos) } \\
\hline $60-69$ & 1,00 & \\
\hline $70-79$ & $0,89(0,73-1,07)$ & 0,217 \\
\hline 80 e mais & $1,05(0,80-1,37)$ & 0,689 \\
\hline \multicolumn{3}{|l|}{ Situação conjugal } \\
\hline Casado/Vive junto & 1,00 & \\
\hline Solteiro/Separado & $0,92(0,73-1,16)$ & 0,505 \\
\hline Viúvo & $0,79(0,64-0,97)$ & 0,027 \\
\hline \multicolumn{3}{|l|}{ Trabalho } \\
\hline Trabalha (aposentado ou não) & 1,00 & \\
\hline Aposentado & $0,75(0,62-0,91)$ & 0,005 \\
\hline Dona de casa & $0,76(0,58-1,00)$ & 0,054 \\
\hline Outros & $0,47(0,26-0,85)$ & 0,013 \\
\hline \multicolumn{3}{|l|}{ Escolaridade (anos de estudo) } \\
\hline $0-3$ & 1,00 & \\
\hline $4-8$ & $1,17(0,92-1,49)$ & 0,175 \\
\hline 9 ou mais & $1,30(0,96-1,75)$ & 0,078 \\
\hline
\end{tabular}

IC95\%: intervalo de 95\% de confiança; RP: razão de prevalências.

* Calculada por meio do modelo final da regressão múltipla de Poisson, considerando todas as variáveis com

valor de $\mathrm{p}$ menor que 0,20 na análise simples.

et al. 25 avaliaram o bem-estar subjetivo e fatores associados em 123 idosos institucionalizados, em João Pessoa, Paraíba. A relação da saúde com a felicidade também tem sido avaliada por meio de pesquisa qualitativa 26 .

Os resultados do presente estudo apontam uma associação inversa entre o sentimento de felicidade por todo o tempo nas últimas quatro semanas e a situação de ser viúvo, o que também foi observado em outras pesquisas 20,27. A importância do cônjuge na idade madura é referenciada principalmente pelo suporte, pela companhia e minimização da solidão 27 . A falta do cônjuge, por sua vez, é um evento estressante, agravandose na população idosa pela maior dificuldade de um novo casamento 20 .

Ter atividade ocupacional associou-se diretamente com o sentimento de felicidade, mesmo após ajuste por outras variáveis. A inserção ocupacional tende a melhorar a autoestima do idoso, o engajamento social e a qualidade dos relacionamentos 28,29. Estudo realizado no Brasil, sobre experiências de felicidade de pessoas idosas, constatou, por meio de análise fenomenológica, que a autonomia e a capacidade para trabalhar proporcionam maior autoestima e bem-estar 26 .

Os comportamentos relacionados à saúde são merecedores de atenção por se associarem com a mortalidade, com a morbidade e com o estado de saúde, sendo ainda fatores de risco passíveis de mudança ${ }^{30}$. No Brasil, políticas para prevenção e controle das doenças crônicas têm focado suas estratégias na promoção de estilos de vida mais saudáveis, especialmente no que se refere ao controle do tabaco, à melhora da dieta, à prática de atividade física e à diminuição do consumo abusivo de bebida alcoólica, além do controle da obesidade 31 . Os resultados deste estudo indicam que o sentimento de felicidade por todo o tempo associa-se significativamente com a ingestão de bebida alcoólica, desde que esta seja ingerida ocasionalmente; com a prática de atividade física no lazer, mesmo sem atingir os padrões recomendados; e com o consumo diário 
Tabela 4

Prevalência e razão de prevalências (RP) do sentimento de felicidade por todo o tempo, em relação a comportamentos relacionados à saúde, na população idosa de Campinas, São Paulo, Brasil. ISA-Camp, 2008.

\begin{tabular}{|c|c|c|c|c|c|c|c|}
\hline Variável & n (\%) & $\begin{array}{l}\text { Prevalência de pessoas } \\
\text { que se declaram felizes } \\
\text { por todo o tempo } \\
\text { (IC95\%) }\end{array}$ & $\begin{array}{l}\text { Valor } \\
\text { de } p \text { * }\end{array}$ & RP bruta (IC95\%) & $\begin{array}{l}\text { Valor } \\
\text { de } p\end{array}$ & $\begin{array}{l}\text { RP ajustada ** } \\
\text { (IC95\%) }\end{array}$ & $\begin{array}{l}\text { Valor } \\
\text { de p }\end{array}$ \\
\hline Hábito de fumar & & & 0,839 & & & & \\
\hline Fumante & $166(11,6)$ & $33,0(24,7-42,6)$ & & 1,00 & & 1,00 & \\
\hline Não fumante & $973(67,3)$ & $35,6(30,3-41,4)$ & & $1,07(0,83-1,38)$ & 0,547 & $1,14(0,89-1,46)$ & 0,261 \\
\hline Ex-fumante & $103(20,9)$ & $35,9(27,7-45,1)$ & & $1,08(0,79-1,49)$ & 0,597 & $1,06(0,77-1,46)$ & 0,697 \\
\hline Ingestão de bebida alcoólica & & & $<0,001$ & & & & \\
\hline Não ingere & $990(68,0)$ & $31,2(26,0-36,9)$ & & 1,00 & & 1,00 & \\
\hline Menos de 1 vez na semana & $299(21,4)$ & $46,3(38,1-54,7)$ & & $1,48(1,20-1,82)$ & $<0,001$ & $1,30(1,06-1,58)$ & 0,011 \\
\hline Mais de 1 vez na semana & $141(10,4)$ & $40,3(30,7-50,8)$ & & $1,29(0,99-1,68)$ & 0,057 & $1,08(0,82-1,42)$ & 0,569 \\
\hline Uso abusivo de bebida alcoólica & & & 0,498 & & & & \\
\hline Não presente & $137(95,6)$ & $35,6(30,6-41,0)$ & & 1,00 & & 1,00 & \\
\hline Presente & $59(04,3)$ & $31,0(19,2-45,9)$ & & $0,87(0,57-1,32)$ & 0,513 & $0,73(0,48-1,10)$ & 0,134 \\
\hline Atividade física no lazer & & & $<0,001$ & & & & \\
\hline Sedentário & $964(66,7)$ & $30,8(26,2-35,8)$ & & 1,00 & & 1,00 & \\
\hline Insuficientemente ativo & $156(11,0)$ & $43,3(33,3-54,0)$ & & $1,40(1,08-1,82)$ & 0,011 & $1,35(1,06-1,70)$ & 0,013 \\
\hline Ativo & $311(22,3)$ & $45,2(36,5-54,2)$ & & $1,46(1,22-1,75)$ & $<0,000$ & $1,37(1,14-1,65)$ & 0,001 \\
\hline $\begin{array}{l}\text { Ingestão de verduras e legumes } \\
\text { todos os dias }\end{array}$ & & & $<0,001$ & & & & \\
\hline Não & $565(39,1)$ & $25,6(19,6-32,8)$ & & 1,00 & & 1,00 & \\
\hline Sim & $866(60,9)$ & $41,7(36,9-46,6)$ & & $1,62(1,28-2,05)$ & $<0,001$ & $1,58(1,28-1,96)$ & $<0,001$ \\
\hline Ingestão de frutas todos os dias & & & 0,005 & & & & \\
\hline Não & $607(41,9)$ & $29,0(22,4-36,6)$ & & 1,00 & & 1,00 & \\
\hline Sim & $824(58,1)$ & $40,0(35,4-45,2)$ & & $1,37(1,08-1,74)$ & 0,009 & $1,31(1,06-1,62)$ & 0,012 \\
\hline $\mathrm{IMC}\left(\mathrm{kg} / \mathrm{m}^{2}\right)$ & & & 0,004 & & & & \\
\hline $22-27$ & $647(45,6)$ & $39,9(33,5-46,7)$ & & 1,00 & & 1,00 & \\
\hline Menor que 22 & $237(16,4)$ & $35,6(29,2-42,6)$ & & $0,89(0,73-1,08)$ & 0,238 & $0,94(0,77-1,14)$ & 0,548 \\
\hline $28-29$ & $265(18,5)$ & $34,5(27,0-42,9)$ & & $0,86(0,68-1,09)$ & 0,227 & $0,87(0,70-1,08)$ & 0,221 \\
\hline 30 ou mais & $282(19,3)$ & $25,4(18,7-33,6)$ & & $0,63(0,48-0,83)$ & 0,002 & $0,69(0,53-0,90)$ & 0,009 \\
\hline Duração do sono (horas) & & & 0,015 & & & & \\
\hline$\leq 5$ & $114(07,9)$ & $26,5(18,9-35,8)$ & & $0,68(0,48-0,98)$ & 0,040 & $0,71(0,49-1,01)$ & 0,062 \\
\hline 6 & $177(12,6)$ & $33,6(26,1-42,1)$ & & $0,87(0,67-1,12)$ & 0,284 & $0,86(0,68-1,10)$ & 0,252 \\
\hline $7-8$ & $795(56,0)$ & $38,6(32,5-45,0)$ & & 1,00 & & 1,00 & \\
\hline 9 & $190(13,3)$ & $38,8(29,6-48,8)$ & & $1,00(0,78-1,28)$ & 0,966 & $1,01(0,79-1,29)$ & 0,885 \\
\hline$\geq 10$ & $142(10,0)$ & $24,0(16,4-33,6)$ & & $0,62(0,43-0,89)$ & 0,011 & $0,65(0,46-0,93)$ & 0,021 \\
\hline Qualidade do sono & & & $<0,001$ & & & & \\
\hline Não dorme mal & $1.029(72,3)$ & $40,0(34,6-45,7)$ & & 1,00 & & 1,00 & \\
\hline Dorme mal & $402(27,6)$ & $23,3(17,4-30,4)$ & & $0,58(0,45-0,74)$ & 0,001 & $0,61(0,48-0,78)$ & $<0,001$ \\
\hline
\end{tabular}

IC95\%: intervalo de 95\% de confiança.

* Teste de qui-quadrado;

** Calculada por meio de regressão múltipla de Poisson. Variáveis incluídas no modelo: sexo, idade, escolaridade, situação conjugal e ocupação.

de legumes, verduras e frutas. Os idosos com IMC elevado, os que mantêm um padrão de sono diário de 10 horas ou mais e os que declaram dormir mal apresentaram-se prejudicados em relação ao sentimento de felicidade.
A associação da prática de atividade física com o maior tempo de felicidade, mesmo nos idosos insuficientemente ativos, poderia ser parcialmente atribuída ao aumento da concentração de triptofano durante o exercício. Trata-se de 
Prevalência e razão de prevalências (RP) do sentimento de felicidade por todo o tempo, em relação ao número de doenças crônicas, à autoavaliação de saúde e à capacidade funcional, na população idosa de Campinas, São Paulo, Brasil. ISA-Camp, 2008.

\begin{tabular}{|c|c|c|c|c|c|c|c|}
\hline Variável & n (\%) & $\begin{array}{l}\text { Prevalência de } \\
\text { pessoas que se } \\
\text { declaram felizes } \\
\text { por todo o tempo } \\
\text { (IC95\%) }\end{array}$ & $\begin{array}{l}\text { Valor } \\
\text { de } p \text { * }\end{array}$ & RP bruta (IC95\%) & $\begin{array}{l}\text { Valor } \\
\text { de } p\end{array}$ & $\begin{array}{l}\text { RP ajustada ** } \\
\text { (IC95\%) }\end{array}$ & $\begin{array}{l}\text { Valor } \\
\text { de } p\end{array}$ \\
\hline Número de doenças crônicas & & & $<0,001$ & & & & \\
\hline 4 ou mais & $234(16,58)$ & $23,4(17,5-30,6)$ & & 1,00 & & 1,00 & \\
\hline 2 ou 3 & $562(39,8)$ & $30,9(25,9-36,5)$ & & $1,32(1,01-1,71)$ & 0,036 & $1,28(0,99-1,66)$ & 0,056 \\
\hline 1 & $337(23,8)$ & $40,2(32,1-48,9)$ & & $1,71(1,27-2,31)$ & 0,001 & $1,56(1,16-2,09)$ & 0,001 \\
\hline Nenhuma & $278(19,7)$ & $49,2(41,1-57,3)$ & & $2,10(1,53-2,88)$ & $<0,001$ & $1,83(1,34-2,51)$ & $<0,001$ \\
\hline Autoavaliação da saúde & & & $<0,001$ & & & & \\
\hline Ruim/Muito ruim & $158(10,8)$ & $08,3(05,0-13,5)$ & & 1,00 & & 1,00 & \\
\hline Boa & $926(64,2)$ & $33,6(27,9-39,9)$ & & $4,02(2,42-6,68)$ & $<0,001$ & $3,74(2,23-6,26)$ & $<0,001$ \\
\hline Excelente/Muito boa & $347(24,8)$ & $51,7(45,1-58,2)$ & & $6,19(3,76-10,1)$ & $<0,001$ & $5,49(3,31-9,11)$ & $<0,001$ \\
\hline Incapacidade funcional & & & $<0,001$ & & & & \\
\hline Grave & $135(0,9)$ & $14,5(07,3-26,6)$ & & 1,00 & & 1,00 & \\
\hline Moderada & $116(0,7)$ & $26,6(17,1-38,8)$ & & $1,83(0,91-3,66)$ & 0,087 & $1,79(0,90-3,55)$ & 0,093 \\
\hline Leve & $352(24,1)$ & $29,7(22,1-38,6)$ & & $2,04(1,06-3,91)$ & 0,031 & $1,95(1,02-3,70)$ & 0,042 \\
\hline Ausente & $828(58,6)$ & $42,3(36,6-48,1)$ & & $2,91(1,58-5,35)$ & 0,001 & $2,52(1,38-4,60)$ & 0,003 \\
\hline
\end{tabular}

IC95\%: intervalo de 95\% de confiança.

* Teste de qui-quadrado;

** Calculada por meio de regressão de Poisson. Variáveis incluídas no modelo: sexo, idade, escolaridade, situação conjugal e ocupação.

aminoácido responsável pela síntese de serotonina, que exerce efeitos no sistema nervoso do indivíduo, promovendo melhora do humor. Peptídeos opióides, particularmente a $\beta$-endorfina, aumentam com o exercício, principalmente naqueles de longa duração e menor intensidade, regulando as catecolaminas e o cortisol 32 . O efeito deste opióide pode desencadear o que se denomina "alegria do exercício", com um aumento da tolerância à dor, controle do apetite e redução da depressão e ansiedade, concomitantemente com o aumento da duração do exercício ${ }^{33}$. Nesse sentido, e possivelmente por outros ainda obscuros, o exercício físico poderia contribuir para melhorar o quadro de sintomas depressivos em idosos; em adição, tem sido eficiente para o tratamento da depressão, conforme expressa a revisão de literatura realizada por Moraes et al. 34 . Por outro lado, estudos têm apontado que os indivíduos mais felizes e otimistas apresentam maiores tendências para apresentar maior nível de atividade física do que aqueles menos felizes 16,22.

A maior prevalência de sentir-se feliz o tempo todo encontra-se presente nos idosos que ingerem bebida alcoólica ocasionalmente, compa- rando-se com aqueles que não a ingerem. Esta associação é conhecida também em relação à capacidade funcional 35 , ao estado de saúde 36 e à autoavaliação de saúde 37 , em estudos realizados com idosos no Brasil. Há evidências de que a ingestão moderada de bebida alcoólica também pode atuar de maneira protetora para algumas doenças cardíacas e em quadros de depressão 38,39. No entanto, é importante considerar que estudos sobre esta relação são mais frequentes em países europeus; o efeito do álcool pode ser considerado benéfico restritamente às pessoas idosas, além de ser evidente em regiões em que o álcool é consumido de maneira regular e moderadamente. Por outro lado, a literatura aponta que o consumo abusivo é prejudicial por ampliar não só o risco de acidentes e violências 40,41, mas também de doenças como cirrose, câncer e doenças cardiovasculares, aumentando as concentrações de lipoproteínas de baixa densidade (LDL), a frequência de oclusão coronariana e o risco de trombose 30,42 .

O consumo de verduras, legumes e frutas associou-se ao sentimento de felicidade nos idosos estudados. São poucas as pesquisas sobre a rela- 
ção do consumo dietético com o bem-estar subjetivo na população idosa. Uma pesquisa com 3.461 adolescentes e jovens latino-americanos detectou que aqueles que consomem verduras e vegetais todos os dias apresentam maior chance de serem classificados como muito felizes 43 . Veenhoven 11 cita uma pesquisa conduzida na Dinamarca, na qual foi observado que as pessoas que sempre ingerem fast food tendem a ser menos felizes. Outros elementos do padrão de vida desses indivíduos, como a falta de tempo e recurso para uma refeição mais saudável, poderiam influenciar essa relação.

Os idosos que se encontram em um padrão diário de sono de 10 horas ou mais e que declaram dormir mal apresentam menor prevalência do sentimento de felicidade por todo o tempo. A importância do sono é reconhecida na literatura por promover a restituição física diária, atuando nos sistemas endócrino, imunológico e metabólico, beneficiando o humor, a atenção e a memória, além de diminuir riscos de acidentes 44,45 . A literatura tem apontado que um padrão de sono diferente de 7 a 8 horas, que é considerado padrão de sono médio, associa-se à maior mortalidade e à maior prevalência de doenças como diabetes, hipertensão 46,47, depressão, doenças reumáticas, osteoporose 48 e obesidade 49 . O padrão de sono, de um lado, é componente de uma escolha, de um estilo de vida; de outro, é afetado pela presença de morbidades e de uso de medicamentos.

A obesidade (IMC $\geq 30 \mathrm{~kg} / \mathrm{m}^{2}$ ) associou-se, neste estudo, inversamente ao sentimento de felicidade por todo o tempo. Alguns estudos relatam resultados semelhantes sobre a relação do sobrepeso e obesidade com a felicidade, o foco, no entanto, é em crianças e adolescentes 50,51. Entre os vários problemas gerados pela obesidade, o aumento do risco de doenças, assim como o comprometimento do sistema locomotor e da autoestima acarretam prejuízos no bem-estar 33 .

O maior tempo de felicidade esteve presente nos idosos com menor número de doenças crônicas. Joia et al. 17 observaram que não apresentar diabetes foi um dos fatores que se associaram à satisfação com a vida na população idosa (odds ratio - OR = 2,63; IC95\%: 1,31-5,27). Estudos internacionais prospectivos evidenciam a importância do otimismo e da vitalidade na proteção à ocorrência de doenças cardíacas. Kubansk \& Thuston 14, com dados do National Health and Nutrition Examination Survey (NHANES I) e o NHANES Epidemiologic Follow-up Study, verificaram que maiores níveis de vitalidade, medidos por meio da Escala Geral de Bem-Estar (GWS), predizem menores riscos de doença coronariana. Kubzansk et al. 13, com um estudo prospectivo de
10 anos, que incluiu 1.306 homens, revelam que indivíduos com sentimento pessimista, medido por meio da Escala Revisada de Otimismo/Pessimismo (PSM-R), apresentaram maiores riscos de angina, infarto não fatal do miocárdio e morte por doença coronariana.

A autoavaliação da saúde como excelente, muito boa ou boa associou-se, de maneira importante, ao sentimento de felicidade por todo o tempo, e estes resultados estão de acordo com os encontrados em estudos prévios, conduzidos com adultos no Brasil 20,21. Barata et al. ${ }^{21}$, estudando a autoavaliação de saúde segundo fatores de vulnerabilidade, discriminação e felicidade, em adultos residentes na área central da cidade de São Paulo, detectaram, entre as três variáveis estudadas, a maior magnitude de associação da autoavaliação do estado de saúde com o grau de felicidade, mesmo na análise ajustada. No presente estudo, igualmente, a associação mais forte, entre as variáveis de condições de saúde, foi com a autoavaliação, em que a prevalência da felicidade por maior tempo esteve presente cinco vezes mais nos idosos que avaliaram sua saúde como excelente ou muito boa, em relação àqueles que avaliaram sua saúde como ruim ou muito ruim. Nos Estados Unidos, estudo longitudinal com 851 indivíduos, que tinham idade média de 73 anos, encontrou que os afetos positivos, referindo-se à felicidade e à vitalidade ou sentimento de energia, foram preditores independentes da autoavaliação de saúde, com maior associação do que os aspectos negativos, como doenças, uso de medicamentos e incapacidades 52 .

A presente pesquisa encontrou forte associação entre a incapacidade funcional e o menor tempo do sentimento de felicidade no idoso. Resultados de um estudo do tipo coorte prospectivo, acompanhando 3.363 pessoas idosas durante aproximadamente oito anos, indicaram que os sentimentos de satisfação com a vida e de felicidade estiveram associados com o menor desenvolvimento de limitações físicas durante o seguimento. É reconhecido que a boa condição de capacidade funcional é indicadora de maior independência e autonomia do idoso, o que o torna capaz de administrar sua própria vida e estar engajado em atividades que são fatores importantes para o bem-estar 29 .

Uma das limitações do presente estudo é que a questão utilizada para avaliar o sentimento de felicidade faz parte de um dos itens que compõem a escala de saúde mental de um instrumento genérico de medida do estado de saúde (SF-36), não tendo sido construída para ser utilizada separadamente da escala. No entanto, tratase de questão única sobre o sentimento de felicidade nas últimas quatro semanas, com cinco 
categorias de respostas; segundo Diener 4, questões com este padrão são eficientes para avaliar a felicidade nos estudos em larga escala. Outros autores também consideram que a frequência e a duração do sentimento de felicidade têm sido consideradas como melhores indicadoras de bem-estar do que a intensidade do sentimento. As pessoas não precisam usufruir de sentimentos intensos de felicidade. Sentir-se feliz por mais tempo promoveria maior referência de percepção de bem-estar 5,6.

Outra limitação é que os estudos do tipo transversal usualmente não permitem identificar fatores causais. É possível que os determinantes e as condições de saúde estudadas estejam agindo sobre o sentimento de felicidade, ou, num sentido inverso, que o sentimento de felicidade atue, propiciando melhores condições de vida e de saúde nos idosos. Sendo assim, o incentivo para um estilo de vida saudável é importante, mas, por outra perspectiva, elevar o nível de saúde mental da população poderia resultar em mudanças comportamentais em relação à saúde.

Na proposta de avaliação do desempenho do sistema de saúde brasileiro (PRO-ADESS) 53, o bem-estar subjetivo foi destacado como uma das subdimensões do estado de saúde que deveria ser aferida. Com base em propostas de outros países, o projeto sugere o uso de indicadores do estado funcional e da autoavaliação de saúde como indicadores de bem-estar. As principais pesquisas nacionais de saúde do Brasil têm incorporado questões de autoavaliação do estado de saúde e/ou de capacidade funcional em seus questionários 54,55. Embora estas questões sejam importantes e estejam relacionadas com o bem-estar, são indicadores que não respondem diretamente à situação de bem-estar subjetivo, que pode ser avaliado por instrumentos específi- $\cos 56$ ou por questão específica relacionada à felicidade ou à satisfação com a vida 4,7 .

A pesquisa identificou que o sentimento de felicidade por todo o tempo esteve presente, em maior parte, nos idosos que ainda trabalham, que ingerem bebida alcoólica moderadamente, nos que são ativos ou insuficientemente ativos no lazer, que consomem frutas, legumes e verduras todos os dias. Os viúvos, os obesos, aqueles que dormem mal e mantêm um padrão de sono de 10 horas ou mais se sentem felizes por menor tempo.

Os indivíduos idosos que não apresentam doenças crônicas ou têm apenas uma doença, em maior parte, sentem-se felizes por todo o tempo, em comparação com aqueles que apresentam quatro doenças ou mais. Os que apresentam incapacidades leves ou não são incapazes em atividades diárias sentem-se mais felizes do que aqueles que apresentam incapacidades graves. Idosos que avaliam sua saúde como boa e principalmente como muito boa ou excelente também fazem parte do segmento dos que se sentem felizes por todo o tempo. As questões de saúde - particularmente os comportamentos, as doenças e incapacidades - e a autoavaliação de saúde tiveram a maior importância na determinação dos fatores associados à felicidade.

Este estudo é um dos primeiros, em base populacional, que estuda as relações das condições de saúde com o bem-estar subjetivo de idosos. Avalia-se que os resultados desta pesquisa possam contribuir para ampliar o conhecimento desse tema no Brasil, apontando a importância de se incorporarem indicadores de bem-estar, entre eles o sentimento de felicidade, na avaliação e no desenvolvimento de programas de atenção à saúde da população idosa. 


\section{Resumo}

O objetivo foi detectar fatores associados à felicidade na população idosa. O estudo é do tipo transversal, de base populacional, com dados de 1.431 idosos do ISA-Camp 2008. A amostragem foi probabilística, por conglomerado e em dois estágios. Foram estimadas as prevalências do sentimento de felicidade por todo o tempo, segundo variáveis sociais, demográficas, de comportamentos e condições de saúde. Os idosos que se sentem felizes por maior tempo são os casados, os que trabalham, são ativos ou insuficientemente ativos no lazer, ingerem bebida alcoólica ocasionalmente, consomem frutas, legumes e verduras todos os dias, não são obesos, apresentam um tempo de sono $<10$ horas e dormem bem. As maiores prevalências do maior tempo de felicidade estão nos idosos que não apresentam doenças, que avaliam melhor a própria saúde e apresentam menos incapacidades. O sentimento de felicidade relaciona-se fortemente com vários indicadores de saúde, sugerindo a adequação do uso complementar do indicador para a avaliação de programas de promoção da saúde de idosos.

Saúde do Idoso; Inquéritos Epidemiológicos; Felicidade

\section{Colaboradores}

M. G. Lima realizou a proposta do artigo, a revisão de literatura, a análise dos dados e a redação do manuscrito. M. B. A. Barros orientou a proposta, análise dos dados e a redação do manuscrito. M. C. G. P. Alves colaborou nas análises estatísticas e revisão do manuscrito.

\section{Agradecimentos}

Os autores agradecem ao Conselho Nacional de Desenvolvimento Científico e Tecnológico (CNPq; processo $\mathrm{n}^{\mathrm{o}}$. 409747/2006-8) pelo financiamento da pesquisa e pela bolsa de produtividade de M. B. A. Barros. Agradecimentos ao Ministério da Saúde e à Secretaria de Saúde de Campinas pelo suporte financeiro para a realização do inquérito (parceria com a UNICAMP/FUNCAMP/ SMS n ${ }^{\circ}$. 4300).

\section{Referências}

1. Evans RG, Stoddart GL. Producing health, consuming health care. Soc Sci Med 1990; 31:1347-63.

2. Brock DW. The separatibility of health and wellbeing. In: Murray CJL, Joshua AS, Mathers CD, Lopez A, editors. Summary measures of population health: concepts, ethics, measurement and applications. Geneva: World Health Organization; 2002. p. 115-20.

3. Minayo MCS, Hartz ZMA, Buss PM. Qualidade de vida e saúde: um debate necessário. Ciênc Saúde Coletiva 2000; 5:7-18.

4. Diener E. Subjective well-being. Psychol Bull 1984; 95:542-75.

5. Vianna FRL. A representação da felicidade no pensamento ocidental [Tese de Doutorado]. Rio de Janeiro: Instituto de Psicologia, Universidade Federal do Rio de Janeiro; 2005.

6. Diener E. Subjective well-being. The science of happiness and a proposal for a national index. Am Psychol 2000; 55:34-43.
7. Veenhoven R. Questions on happiness. In: Veenhoven R, editor. Happiness in nations. Studies in socio-cultural transformation. Rotterdam: Erasmus University; 1992. p. 28-42.

8. Campbell A. Subjective measures of well-being Am Psycol 1976; 31:117-24.

9. Institute of Medicine. Leading health indicators for healthy people 2020: letter report. Washington DC: The National Academies Press; 2011.

10. Giltay EJ, Geleijnse JM, Zitman FG, Hoestra T, Schouten EG. Dispositional optimism and allcause and cardiovascular mortality in a prospective cohort of elderly dutch men and women. Arch Gen Psychiatry 2004; 61:1126-35.

11. Veenhoven R. Health happiness: effects of happiness on physical health and the consequences for preventive care. J Happiness Stud 2008; 9:449-69.

12. Sadle ME, Miller CJ, Christsen K, McGue M. Subjective wellbeing and longevity: a co-twin control study. Twin Res Hum Genet 2011; 14:249-56. 
13. Kubzansk LD, Sparrow D, Vokonas P, Kawachi I. Is the glass half empty or half full? A prospective study of optimism and coronary heart disease in the Normative Aging Study. Psychosomc Med 2001; 63:910-6.

14. Kubansk LD, Thuston RC. Emotional vitality and incident coronary heart disease. Arch Gen Psychiatry 2007; 64:1393-401.

15. Collins AL, Goldman N, Rodriguez G. Is positive well-being protective of mobility limitations among older adults? J Gerontol B Psycol Soc Sci 2008; 63:321-7.

16. Baruth M, Lee DC, Sui X, Church TS, Marcus BH, Wicox S, et al. Emotional outlook on life predicts increases in physical activity among initially inactive men. Health Educ Behav 2011; 38:150-8.

17. Joia CL, Ruiz T, Donalisio MR. Condições associadas ao grau de satisfação com a vida entre a população de idosos. Rev Saúde Pública 2007; 41:131-8.

18. Portero CF. Social support, psycological well-being, and health among the elderly. Educ Gerontol 2007; 33:1053-68.

19. Tuntichaivanit C, Nanthamongkolchai S, Munsawaengsub C, Charupoonphol P. Life happiness of the elderly in Rayong Province. J Public Health 2009; 39:34-47.

20. Rodrigues A, Silva JA. O papel das características sociodemográficas na felicidade. Psico USF 2010; 15:113-23.

21. Barata RB, Ribeiro MCSA, Cassanti AC; Grupo do Projeto Vulnerabilidade Social no Centro de São Paulo. Social vulnerability and health status: a household survey in the central area of a Brazilian metropolis. Cad Saúde Pública 2011; 27 Suppl 2:S164-75

22. Albarracin D, Hart W. Positive $\operatorname{mood}+$ action $=$ negative mood + inaction: effects if general action and inaction concepts on decisions and performance as a function of affect. Emotion 2011; 11:951-7.

23. Ciconelli RM, Ferraz MB, Santos W, Meinão I, Quaresma MR. Tradução para a língua portuguesa e validação do questionário genérico de avaliação de qualidade de vida SF-36 (Brasil SF-36). Rev Bras Reumatol 1999; 39:143-50.

24. Barbor TE, La Fuente JR, Saunders J, Grant M. AUDIT - The Alcohol Use Disorders Identification test: guidelines for use in primary health care. Geneva: World Health Organizaton; 1992.

25. Guedea MTD, Albuquerque FJB, Tróccoli BT, Noriega JAV, Seabra MAB, Guedea RLD. Relação do bem-estar subjetivo, estratégias de enfrentamento e apoio social em idosos. Psicol Reflex Crit 2005; 19:301-8.

26. Luz MMC, Amatuzzi MM. Vivências de felicidade de pessoas idosas. Estud Psicol (Campinas) 2008; 25:303-7.

27. George LK. Still happy after these years: research frontiers on subjective well-being in later life. J Gerontol B Psychol Sci Soc Sci 2010; 65B:331-9.

28. Veras RP, Ramos LR, Kalache A. Crescimento da população idosa no Brasil: transformações e consequências na sociedade. Rev Saúde Pública 1987; 21:225-33.
29. Deps VL. Atividade e bem-estar psicológico na maturidade. In: Neri AL, organizador. Qualidade de vida e idade madura. 7a Ed. Campinas: Editora Papirus; 2007. p. 57-82.

30. World Health Organization. Global health risks: mortality and burden of disease attributable to selected major risks. Geneva: World Health Organization; 2009.

31. Departamento de Análise de Situação de Saúde, Secretaria de Vigilância em Saúde, Ministério da Saúde. Plano de ações estratégicas para o enfrentamento das doenças crônicas não transmissíveis (DCNT) no Brasil 2011-2022. Brasília: Ministério da Saúde; 2011.

32. Stella SG, Antunes HKM, Santos RF, Galduróz JCF, Mello MT. Transtornos do humor e exercício físico. In: Mello MT, Tufik S, organizadores. Atividade física, exercício físico e aspectos psicobiológicos. Rio de Janeiro: Editora Guanabara Koogan; 2004. p. 51-9.

33. McArdle WD, Katch FI, Katch VL. Sistema endócrino: organização e respostas agudas e crônicas ao exercício. In: McArdle WD, Katch FI, Katch VL, organizadores. Fisiologia do exercício-energia, nutrição e desempenho humano. 6a Ed. Rio de Janeiro: Editora Guanabara Koogan; 2008. p. 419-64.

34. Moraes H, Deslandes A, Ferreira C, Pompeu FAMS, Ribeiro P, Laks J. O exercício físico no tratamento da depressão em idosos: revisão sistemática. Rev Psiquiatr Rio Gd Sul 2007; 29:70-9.

35. Santos JLF, Lebrão ML, Duarte YAO, Lima FD. Functional performance of the elderly in instrumental activities of daily living: an analysis in the municipality of São Paulo, Brazil. Cad Saúde Pública 2008; 24:879-86.

36. Lima MG, Barros MBA, César CLG, Goldbaum M, Carandina L, Alves MCG. Health-related behavior and quality of life among the elderly: a populationbase study. Rev Saúde Pública 2011; 45:485-93.

37. Borim FSA, Barros MBA, Neri AL. Autoavaliação da saúde em idosos: pesquisa de base populacional no Município de Campinas, São Paulo, Brasil. Cad Saúde Pública 2012; 28:769-80.

38. Klatsky AL. Moderate drinking and reduced risk of heart disease. Alcohol Res Health 1999; 23:15-24.

39. Rodgers B, Korten AE, Jorm AF, Christensen H, Henderson S, Jacomb PA. Risk factors for depression and anxiety in abstainers, moderate drinkers and heavy drinkers. Addiction 2000; 95:1833-45.

40. Rehm J, Mathers C, Popova S, Thavorncharoensap M, Teerawattananon Y, Patra J. Global burden of disease and injury and economic cost attributable to alcohol use and alcohol-use disorders. Lancet 2009; 373:2223-33.

41. Zaridze D, Brennan P, Borehan J, Boroda A, Karpov R, Lazarev A, et al. Alcohol and cause-specific mortality in Russia: a retrospective case-control study of 48557 adult deaths. Lancet 2009; 373:2201-14.

42. McKee M, Britton A. The positive relationship between alcohol and heart disease in eastern Europe: potential physiological mechanisms. J R Soc Med 1999; 91:402-7. 
43. Piqueras JA, Kuhne W, Vera-Villarrol P, Straten AV, Cuijpers P. Happiness and health behaviours in Chile college students: a cross-sectional survey. BMC Public Health 2011; 11:443.

44. Birchler-Pedross A, Schröder CM, Münch M, Knoblauch V, Blatter K, Schnitzler-Sack C, et al. Subjective well-being is modulated by circadian phase, sleep pressure, age and gender. J Biol Rhythms 2009; 24:232-42.

45. Akerstedt TA, Nilsson PM. Sleep as restitution: an introduction. J Intern Med 2003; 254:6-12.

46. Cappuccio FP, D’Elia L, Strazzullo P, Miller MA. Sleep duration and all-cause mortality: a systematic review and meta-analysis of prospective studies. Sleep 2010; 33:585-92.

47. Nagai M, Hoshide S, Kario K. Sleep duration as a risk factor for cardiovascular disease: a review of a recent literature. Curr Cardiol Rev 2010; 6:54-61.

48. Lima MG, Francisco PMSB, Barros M. Sleep duration pattern and chronic disease in Brazilian adults (ISACAMP 2008/2009). Sleep Med 2012; 13:139-44.

49. Patel SR, Malhotra A, White DP, Gottlieb DJ, Hu FB. Association between reduced sleep and weight gain in women. Am J Epidemiol 2006; 164:947-54.

50. Erickson SJ, Robinson TN, Haydel KF, Killen JD. Are overweight children unhappy?: body mass index, depressive symptoms, and overweight concerns in elementary school children. Arch Pediatr Adolesc Med 2000; 154:931-5.
51. Needham BL, Crosnoel R. Overweight status and depressive symptoms during adolescence. J Adolesc Health 2005; 36:48-55.

52. Benyamini $\mathrm{Y}$, Idler EL, Leventhal H, Leventhal EA. Positive affect and function as influences on selfassessments of health: expanding our view beyond illness and disability. J Gerontol B Psychol Sci Soc Sci 2000; 55:P107-16.

53. Almeida C. Projeto: desenvolvimento de metodologia de avaliação do desempenho do sistema de saúde brasileiro (PRO-ADESS). http://www.pro adess.cict.fiocruz.br/relatoriofinal.pdf (acessado em 23/Nov/2011).

54. Travassos C, Viacava F, Laguardia J. Os Suplementos Saúde na Pesquisa Nacional por Amostra de Domicílios (PNAD) no Brasil. Rev Bras Epidemiol 2008; 11 Suppl 1:98-112.

55. Secretaria de Vigilância em Saúde, Ministério da Saúde. VIGITEL 2007. Vigilância de fatores de risco e proteção para doenças crônicas por inquérito telefônico. Brasília: Ministério da Saúde; 2009.

56. McDowell I. Measuring health: a guide to rating scales and questionnaires. $3^{\text {rd }}$ Ed. New York: Oxford University Press; 2006.

Recebido em 03/Abr/2012

Versão final reapresentada em 22/Jul/2012

Aprovado em 07/Ago/2012 